\title{
Effect of electron irradiation on transparent conductive films ZnO:Al deposited at different sputtering power
}

\author{
D.V. Myroniuk ${ }^{1}$, A.I. Ievtushenko ${ }^{1}$, G.V. Lashkarev ${ }^{1}$, V.T. Maslyuk ${ }^{2}$, I.I. Timofeeva ${ }^{1}$, V.A. Baturin ${ }^{3}$, \\ O.Yu. Karpenko ${ }^{3}$, V.M. Kuznetsov ${ }^{3}$, M.V. Dranchuk ${ }^{1}$ \\ ${ }^{1}$ Frantsevich Institute for Problems of Material Science, NAS of Ukraine, \\ 3, Krzhizhanovsky str., 03680 Kyiv, Ukraine \\ ${ }^{2}$ Institute of Electron Physics, NAS of Ukraine, 21, Universitetska str., 88017 Uzhgorod, Ukraine, \\ ${ }^{3}$ Institute of Applied Physics, NAS of Ukraine, 58, Petropavlivska str., 40000 Sumy, Ukraine \\ Corresponding author. Phone: 38(044) 424-15-24; e-mail: denysmyroniuk@gmail.com
}

\begin{abstract}
Transparent conductive oxide thin films of Al-doped $\mathrm{ZnO}$ grown by $\mathrm{rf}$ magnetron sputtering were irradiated with high energy electrons with the energy 12.6 MeV and fluence $5 \cdot 10^{14} \mathrm{e} / \mathrm{cm}^{2}$. The films were produced using different sputtering powers. It has been shown that electron irradiation creates defects that lead to distortions of the crystal lattice, which results in reduced crystallinity of the films. Also, it leads to film heating that results in radiation annealing and relaxation of the lattice.
\end{abstract}

Keywords: transparent conductive aluminium doped zinc oxide, AZO, electron irradiation, point defects, X-ray diffraction, stress, resistance.

Manuscript received 05.03.15; revised version received 28.05.15; accepted for publication 03.09.15; published online 30.09.15.

\section{Introduction}

Transparent conductive film materials have wide applications in heat mirrors, optoelectronic devices, gas sensors, reflecting coatings on windows [1]. Some types of solar cells for space applications require transparent electrodes that should be resistant to ionizing radiation [2].

$\mathrm{ZnO}$ is used as a window, component of heterojunction and electrode layer for thin film solar cells based on ternary photovoltaic materials $\left(\mathrm{CuInSe}_{2}\right.$, $\mathrm{CuInS}_{2}$ ). Besides, the main requirements of high transparency and low resistivity, the mechanical properties are of importance, too. It is necessary to underline that a stress appears due to differences in lattice periods and thermal coefficients of their linear expansion (TCLE), which influence on their structural and electrical parameters. Ohler et al. [3] have shown that the lattice mismatch at InAs/GaAs and InAs/AlAs heterojunctions significantly influences the band offsets. They lead to different electronic properties of this heterojunction. Ellmer et al. [4] have shown that mechanical stresses in $\mathrm{ZnO}$ films decrease the efficiency of solar cells based on $\mathrm{CuInS}_{2}$.

Information about the electron irradiation effect on the crystal structure and electrical properties of $\mathrm{ZnO}$ based materials is very limited, particularly for transparent conductive films. Most publications are devoted to researches of intrinsic defects in bulk $\mathrm{ZnO}$ [57]. Due to particle irradiation, information about the energy of formation of defect levels and their quantity is obtained with the capacitance transient measurements and positron annihilation spectroscopy $[8,9]$.

However, recently the devices based on zinc oxide films acquired rather widespread use. Gur et al. [10] investigated changes of structure properties under the 
influence of electron radiation, they found that electron irradiation causes partial recrystallization of the films and generate intrinsic defect interstitial $\mathrm{Zn}$. But they have not yet produced sufficient information about electron irradiation effect on properties of the $\mathrm{ZnO}$ films doped with aluminum. Since the deposition parameters strongly influence on the structure and electrical properties, the effect of irradiation should be discussed together with the deposition parameters. This study is an attempt to enlighten the electron irradiation effects on structural and electrical properties of $\mathrm{ZnO}: \mathrm{Al}$ films grown at different magnetron sputtering powers. Also, we have investigated how the power of sputtering affects the radiation interaction with $\mathrm{ZnO}$ films.

\section{Experimental details}

Transparent conductive oxides $\mathrm{ZnO}$ doped with $\mathrm{Al}$ ( $\mathrm{ZnO}: \mathrm{Al}$ ) were deposited by radio frequency (RF) magnetron sputtering (MS) on silicon substrates. The disk of metal zinc (purity 99.99\%) with aluminum inserts (purity $99.99 \%$ ) has been used as a composite target. All inserts were made in the erosion area and covered the 0.02 part of its square. The high-resistant silicon of (100) orientation and $p$-type conductivity was used as a substrate. Its temperature was maintained at $300{ }^{\circ} \mathrm{C}$. The target-substrate distance was kept constant at $70 \mathrm{~mm}$. The vacuum chamber was filled with argon and oxygen at partial pressures 1 and $0.05 \mathrm{~Pa}$, respectively. The magnitudes of sputtering power were 140, 170 and $200 \mathrm{~W}$. To improve the crystalline perfection of the AZO films, we used the special technological approach in magnetron sputtering. After deposition of the first layer, the process was interrupted for $2 \mathrm{~min}$, i.e., we used start-stop technique $[11,12]$. It provides good growth conditions and prevents overheating the deposited layer. All films were grown using these three start-stop stages. The total sputtering time was $6 \mathrm{~min}$. The thicknesses of the films were measured using ex-situ ellipsometry on duplicated samples deposited on glass substrates.

The films were irradiated by electrons with high energy about $12.6 \mathrm{MeV}$ and the fluence $5 \cdot 10^{14} \mathrm{e} / \mathrm{cm}^{2}$ using the microtron electron accelerator $\mathrm{M}-30$ at the Institute of Electron Physics, NAS of Ukraine, Uzhgorod. This fluence can be obtained by an electronic device moving in the Van Allen radiation inner belt for 10 years.

The features of films crystal structure were studied using the XRD analysis where the $\mathrm{CuK} \alpha_{1}$ radiation was used as a source $(\lambda=0.154056 \mathrm{~nm})$. The XRD measurements were carried out by the DRON-4 Powder Diffraction system; a scan rate of $1 \%$ min and a step size of $0.01^{\circ}$ were used. It is important to note that the analyzed surface is about $\sim 1 \mathrm{~cm}^{2}$ and the penetration depth of X-rays at $20-40 \mathrm{keV}$ is several micrometers. The measurement error of the peak position was about $\pm 0.01^{\circ}(2 \theta)$. The error of the $c$-lattice period determination was close $\pm 0.0003 \mathrm{~nm}$. The relative error of full width at half maximum (FWHM) calculations was estimated to be $\pm 2 \%$.

The electrical resistance of zinc oxide films was measured using the four-point probe at room temperature.

\section{Results and discussion}

Fig. 1 shows the results of X-ray diffraction for asgrown and irradiated $\mathrm{ZnO}$ :Al films grown under various sputtering powers. There is only one maximum at $2 \theta=34.4^{\circ}$ that corresponds to diffraction from the $(002)$ planes of wurtzite $\mathrm{ZnO}$ crystal lattice having preferred $c$ axis orientation of crystallites: $c$-axes are predominantly aligned perpendicular to the substrate. There are no diffraction reflections that correspond to the $\mathrm{Al}_{2} \mathrm{O}_{3}$ phase or other ones.

The thicknesses and, consequently, the deposition rate of the films are slightly different at various sputtering powers. The thickness grows from 89 to $108 \mathrm{~nm}$ by the power increase from 140 to $170 \mathrm{~W}$, and then falls to $81 \mathrm{~nm}$ at the sputtering power $200 \mathrm{~W}$.

Fig. 2a shows the increase of the maximum reflection intensity (002) peak for as-grown samples, when the sputtering power enlarges from 140 up to $170 \mathrm{~W}$. Then the maximum intensity decreases sharply at the power $200 \mathrm{~W}$. The higher diffraction peak intensity means the higher degree of film crystallinity. But the ratio of the maximum intensity to the thickness of film keeps its shape curves dependence on the sputtering power (Fig. 2b). The increase of ratio at $170 \mathrm{~W}$ may be attributed to the enhancement of lattice distortions produced by energetic ions $\mathrm{Zn}, \mathrm{O}$ and $\mathrm{Al}$ impinging onto the growing film, i.e., the peening effect $[13,14]$. Peening phenomenon would occur in a crystalline film during the sputtering process [13]. Increasing the sputtering pressure causes thermalization of the energetic particles with working gases ions, and an atomic peening process no longer persists [15]. Further decreasing the intensity maximum, as mentioned in [16], can be explained by several factors which dominate at $P=200 \mathrm{~W}: 1)$ the deposition rate increases and gives newly arrived ions less time to move to their stable sites for epitaxial growing; 2) the ratio $\mathrm{Zn}$ to $\mathrm{ZnO}$, which arrive to a substrate, is enhanced with increasing the power [17], this structure is more crystallographically disordered than if the arriving $\mathrm{Zn}$ were already oxidized; 3 ) the energy of secondary electrons emitted from the target during the sputtering process increases with the target voltage, hence the power increases; 4) another explanation can be found in [18]. The power increase at a constant gas pressure generates surplus metallic $\mathrm{Zn}$ with higher energies on the substrate. Then, $\mathrm{Zn}$ desorbs from the substrate and, consequently, heats the substrate [19]. Furthermore, the Al doping level increases, when the substrate temperature is raised, since the highly reactive $\mathrm{Al}$ does not desorb [18]. 


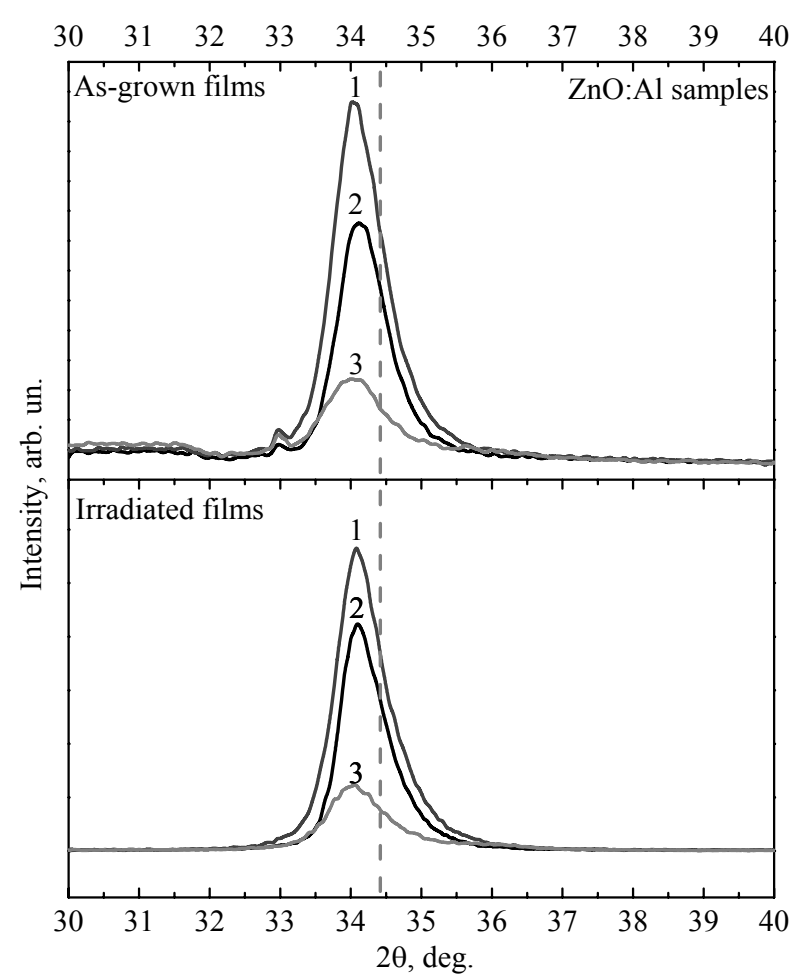

Fig. 1. X-ray patterns for the as-grown and irradiated with electrons $\mathrm{ZnO}$ :Al films deposited at various sputtering powers. Sputtering power, W: 170 (1), 140 (2), 200 (3).

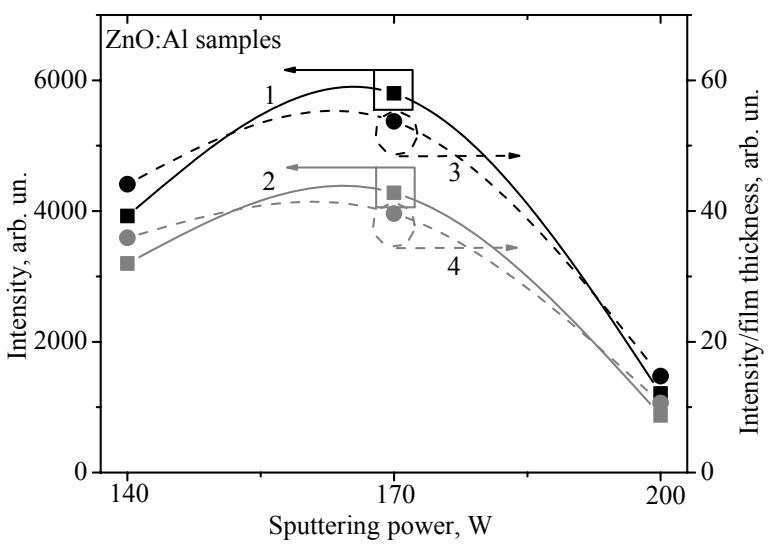

Fig. 2. The dependence of the intensity (002) maximum and maximum relate to film thickness on the sputtering power for the as-grown $(1,3)$ and irradiated $(2,4)$ films.

The maximum intensity of the (002) reflections for films decrease after irradiation in comparison with the as-grown ones (Fig. 2). This phenomenon can be explained by a defect generation.

Most of the energy losses at high energy electron bombardment are caused by electron-electron collisions, rather than the electron-nucleus ones [6]. If a relativistic electron of energy $E$ makes a direct hit on a nucleus, it transfers the maximum energy $E_{m}(\mathrm{eV})$ given by

$$
E_{m}=\frac{2 E\left(E+2 m_{e} c^{2}\right)}{M c^{2}}=\frac{2.147 \cdot 10^{-9} E\left(E+1.022 \cdot 10^{6}\right)}{A}
$$

where $m_{e}$ and $M$ are the electron and ion masses, respectively, $A$ is the atomic weight. The threshold energy $E_{\text {th }}$ necessary to produce an atomic displacement is given by the condition $E_{m}=E_{d}$, where $E_{d}$ is the displacement energy. The electron-nucleus collisions create defects and their clusters, which lead to distortions of the crystal lattice.

For more information on the electrons irradiation impact on the crystal structure depending on the sputtering power, we have calculated X-ray diffraction parameters of as-grown and irradiated films.

From Fig. 3 we can see that the values of the lattice period $c$ of the as-grown samples as well as of irradiated ones are significantly larger than the reference value of the strain-free bulk lattice period $\left(c_{0}=0.5205 \mathrm{~nm}\right)$. The $c$-period values of the films grow with increasing the sputtering power. It implies that all the films have compressive stresses in the film plane.

Calculations of the film stress are based on the biaxial strain model. The strain along the $c$ axis can be expressed as follows:

$\varepsilon=\left(c-c_{0}\right) / c_{0}$.

To derive the film stress $\sigma$ parallel to the film surface (in xy plane), the following formula has been used, which is valid for a hexagonal lattice:

$\sigma=\frac{2 C_{13}^{2}-C_{33}\left(C_{11}+C_{12}\right)}{2 C_{13}^{2}} \cdot \frac{c-c_{0}}{c_{0}}$.

For the elastic constants $C_{i i}$, data of single crystalline $\mathrm{ZnO}$ have been used: $C_{11}=208.8, \quad C_{33}=213.8$, $C_{12}=119.7, C_{13}=104.2 \mathrm{GPa}$. It yields the following numerical equation for the stress derived from XRD:

$\sigma=-223 \cdot \varepsilon[\mathrm{GPa}]$.

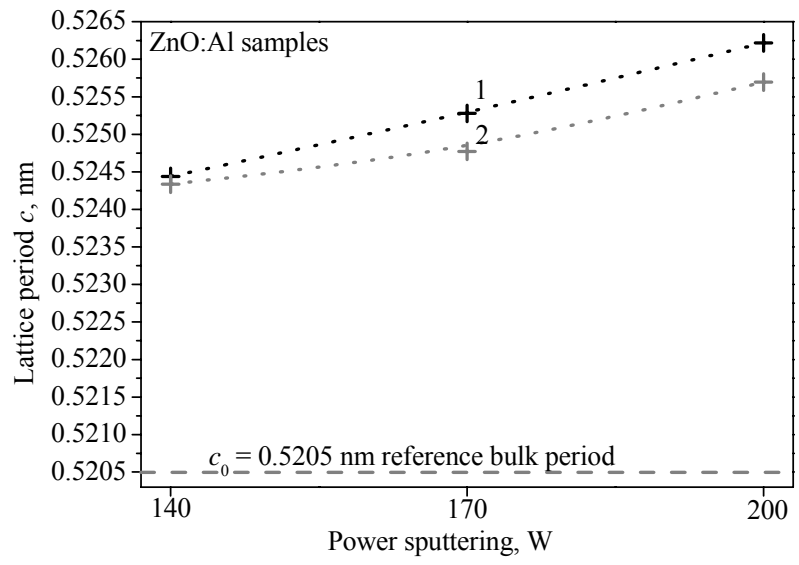

Fig. 3. The effect of the sputtering power on the lattice period $c$ of the as-grown (1) and electron irradiated (2) $\mathrm{ZnO}$ :Al films. 
Fig. 4 shows the development of the film stress with increasing the sputtering power. The compressive stresses in the film plane arise from differences of lattice periods between $\mathrm{ZnO}$ and $\mathrm{Si}$ (100) ( 40\% [20]), TCLE $\alpha$ and the appearance of internal stresses in the film during the growth process.

The difference in TCLE $\alpha_{\mathrm{Si}}=2.6 \cdot 10^{-6} /{ }^{\circ} \mathrm{C}$ and $\alpha_{\mathrm{ZnO} \perp}=4.75 \cdot 10^{-6} /{ }^{\circ} \mathrm{C}$ causes tensile stresses in the film plane in the samples cooled from the deposition temperature down to the room one. The strain in the films along the $c$ axis is of the order of $+0.5 \ldots+1.2 \%$, from which the compressive stress in grains of up to 2.6 GPa occurs (Fig. 4). The thermal strain introduced by the different TCLE is significantly lower $\left(<5 \cdot 10^{-4}\right)$ than measured strain. It shows that the measured film stress is mainly caused by the growth process itself [14]. The intrinsic stress is caused by the accumulating effect of the crystallographic flaws, which are built into the film during its growth [21]. The compressive stresses are believed to be due to energetic argon and oxygen ions that can be sufficiently energetic to penetrate into and become buried in the intergrain space of the growing film. Another reason is that argon and oxygen atoms produce the stresses by being trapped into the grains of films [22]. While the high compressive stress values at low sputtering power can be explained by the atomic peening process as mentioned above (in the case of intensity growth), the compressive stress that occurs in $\mathrm{ZnO}$ at high sputtering power needs another explanation. According to our opinion, the deposition power increase gives less time to additional new atoms to incorporate into film and move to stable site, which is cause of disordering and an appearance of the compressive stress.

After irradiation, the dependence of the $c$ period versus the sputtering power repeats the growth tendency of the as-grown ones (Fig. 3), but the lattice periods reduce and are close to those of the unstrained bulk crystal (Fig. 3). Thus, the compressive stresses of irradiated films decrease (Fig. 4). A similar phenomenon is observed when the samples are annealed [21]. So, we can summarize that electron-electron collisions lead to film heating, which results in radiation annealing and relaxation of the lattice.

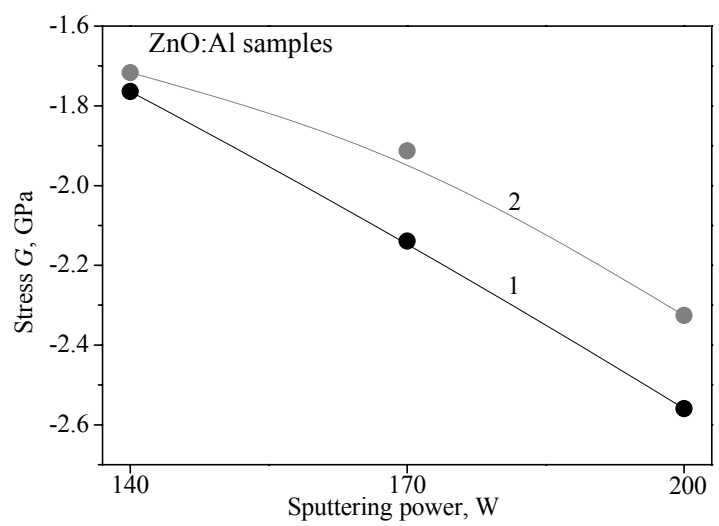

Fig. 4. The stress dependence on the sputtering power of the as-grown (1) and irradiated (2) $\mathrm{ZnO}: \mathrm{Al}$ films.
The values of the full width at half maximum (FWHM) of the diffraction peak (002) for as-grown and irradiated films are presented in Fig. 5. The lower values of FWHM can indicate better crystallinity of the films.

The FWHM values for the as-grown films increase as the sputtering power increases and are consistent with the foregoing considerations. After irradiation, FWHM of reflections almost do not change relatively to the asgrown ones, except the case of the first sputtering power (140 W).

Let us consider the size of coherent scattering regions (CSR) films at all stages. The average grain size in films can be estimated by Scherrer's formula using the FWHM value of the XRD diffraction peaks as follows [23]:

$D=\frac{0.9 \lambda}{\beta \cos \theta}$

where $D, \lambda, \theta$, and $\beta$ are the mean grain size, $\mathrm{X}$-ray wavelength of $0.154 \mathrm{~nm}$, Bragg diffraction angle, and FWHM of the (002) diffraction peak, respectively. Fig. 6 shows that dimensions of CSR for the as-grown samples decrease with the sputtering power increase. The electron irradiation weakly influences on CSR dimension, except the case of low sputtering power at $140 \mathrm{~W}$.

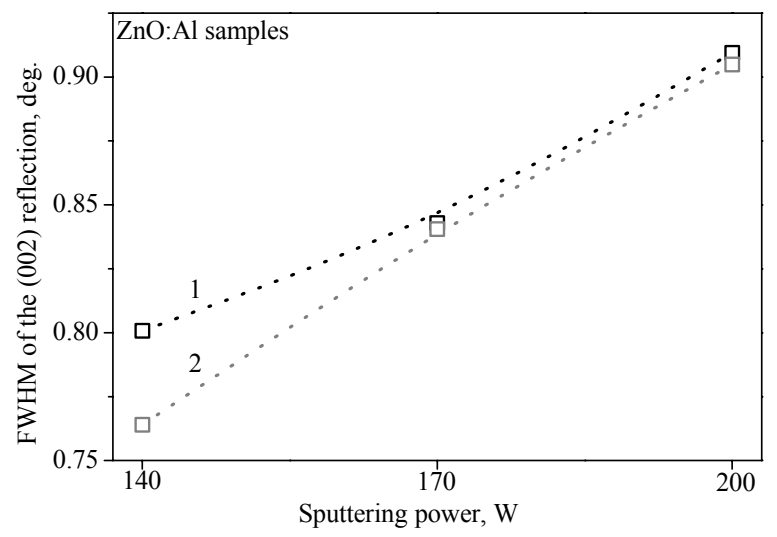

Fig. 5. FWHM of the (002) reflection dependence on the sputtering power for the as-grown (1) and irradiated (2) $\mathrm{ZnO}: \mathrm{Al}$ films.

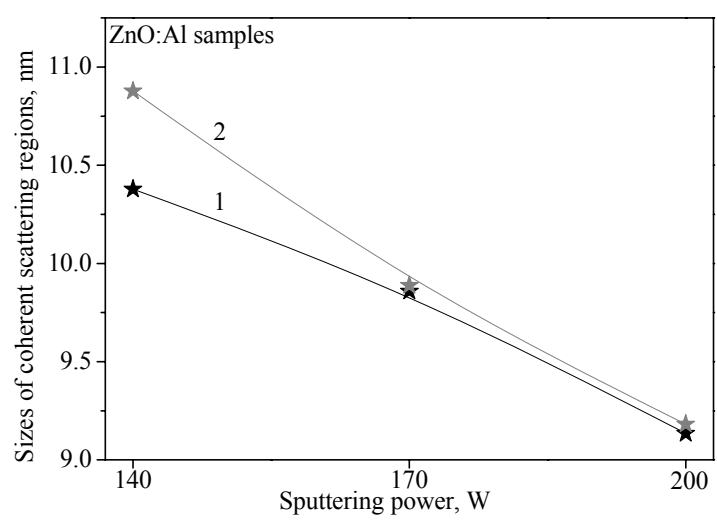

Fig. 6. The dependence of CSR sizes on the sputtering power of the as-grown (1) and irradiated (2) $\mathrm{ZnO}: \mathrm{Al}$ films.

(C) 2015, V. Lashkaryov Institute of Semiconductor Physics, National Academy of Sciences of Ukraine 
The results of electrical measurements are shown in Fig. 7. The film resistance of the as-grown samples increases with the sputtering power from 140 up to $170 \mathrm{~W}\left(3.3 \cdot 10^{-2} \ldots 4.6 \cdot 10^{-2} \Omega \cdot \mathrm{cm}\right)$. At the power $200 \mathrm{~W}$, the resistance decreases sharply $\left(4.7 \cdot 10^{-2} \Omega \cdot \mathrm{cm}\right)$ (Fig. 7). Our results are consistent with those in [24], where it was ascertained that film resistivity increases dramatically reciprocally to the film thickness, if it is less than approximately $100 \mathrm{~nm}$. This dependence is irrespective of the used deposition technique [24]. The data on the resistance partially correlate with the maximum intensities of (002) reflections for films deposited at different sputtering powers. The film obtained at $140 \mathrm{~W}$ has the lowest strain values and, consequently, the resistance is low, as mentioned in [15]. The film at the $170-\mathrm{W}$ power causes the peening process. Oxygen incorporated into the film reacts with $\mathrm{Al}$ and generates $\mathrm{Al}_{2} \mathrm{O}_{3}$, which causes an increase of resistivity due to diminishing the mobility at grain boundaries. It is seen that the almost equal resistivity is obtained with the 200-W sputtering power, when $\mathrm{Zn}$ desorption occurs and $\mathrm{Al}$ doping level increases. The carrier mobility decreases, but the carrier concentration due to $\mathrm{Al}$ doping rises rapidly. Therefore, the electrical resistance of the film does not increase [25].

After irradiation, the electrical resistance of films with higher crystallinity increases as shown in Fig. 7 (at $P<200 \mathrm{~W}$ ). Since irradiation was performed in a familiar environment, (air atmosphere) the film probably adsorbs oxygen from atmosphere. Both the carrier density and mobility are affected by the oxidation processes either in grains (oxygen heals the donor levels of oxygen vacancy) or in intergrain regions (oxidation increases the height of potential barriers between grains). Another reason of increasing resistivity is generation of defects. The films with better crystallinity are stronger affected by irradiation than those obtained at $200 \mathrm{~W}$.

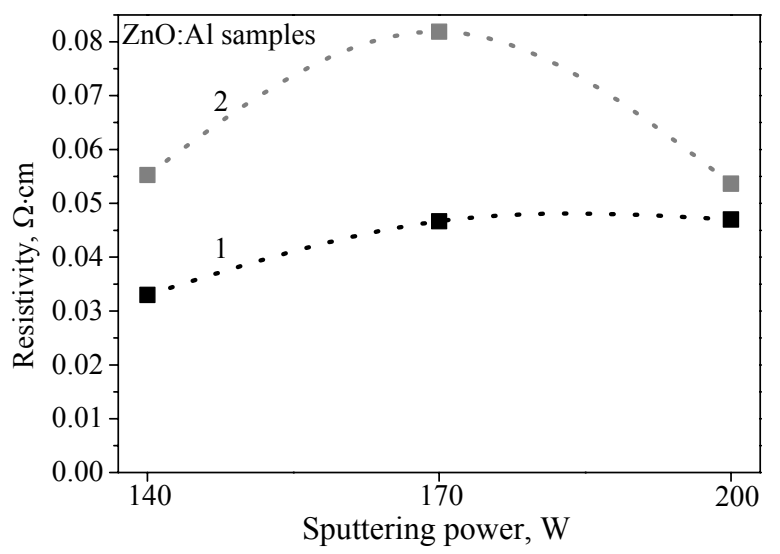

Fig. 7. The resistance dependence on the sputtering power for the as-grown (1) and irradiated (2) $\mathrm{ZnO}: \mathrm{Al}$ films.

\section{Conclusions}

Summarizing, we have reported on the effect of electron irradiation on the properties of $\mathrm{ZnO}$ films deposited at different sputtering powers. The microstructure and electrical properties of $\mathrm{ZnO}$ film are changed by electron bombardment. It was revealed that this type of irradiation effects on the structure, resulting in radiation annealing and creation of defects. The better crystalline quality influences only partially on the resistivity. The electrical activity and quantity of Al impurity, oxidation state (i.e. the stoichiometry of the $\mathrm{ZnO}$ films) are important factors, too.

\section{References}

1. E. Marla, T. Malave-Sanabria, P. Hambrouger et al., Transparent Conducting Thin Films for Spacecraft Applications. NASA Technical Report Server (NTRS), 1994.

2. A. Jasenek, U. Rau, Defect generation in $\mathrm{Cu}(\mathrm{In}, \mathrm{Ga}) \mathrm{Se}_{2}$ heterojunction solar cells by highenergy electron and proton irradiation // J. Appl. Phys. 90(2), p. 650-658 (2001).

3. C. Ohler, A. Förster, J. Moers, C. Daniels, H. Lüth, Band offsets at heavily strained III-V interfaces // J. Phys. D. Appl. Phys. 30(10), p. 1436-1441 (1999).

4. K. Ellmer, R. Cebulla, R. Wendt, Polycrystalline $\mathrm{ZnO}-$ and $\mathrm{ZnO}$ :Al-layers: Dependence of film stress and electrical properties on the energy input during the magnetron sputtering deposition // MRS Proc. 472, p. 245 (1997).

5. D.C. Look, D.C. Reynolds, J.W. Hemsky, R.L. Jones, J.R. Sizelove, Production and annealing of electron irradiation damage in $\mathrm{ZnO} / /$ Appl. Phys. Lett. 75(6), p. 811 (1999).

6. D. Look, J. Hemsky, J. Sizelove, Residual native shallow donor in ZnO // Phys. Rev. Lett. 82, p. 2552-2555 (1999).

7. C. Coskun, D.C. Look, G.C. Farlow, J.R. Sizelove, Radiation hardness of $\mathrm{ZnO}$ at low temperatures // Semicond. Sci. Technol. 19, p. $752-754$ (2004).

8. F.D. Auret, S.A. Goodman, M. Hayes, M.J. Legodi, H.A. van Laarhoven, D.C. Look, Electrical characterization of $1.8 \mathrm{MeV}$ proton-bombarded ZnO // Appl. Phys. Lett. 79(19), p. 3074-3076 (2001).

9. F. Tuomisto, K. Saarinen, D.C. Look, Irradiationinduced defects in $\mathrm{ZnO}$ studied by positron annihilation spectroscopy // physica status solidi (a), 201(10), p. 2219-2224 (2004).

10. E. Gür, H. Asıl, C. Coşkun et al., Optical and structural properties of $\mathrm{ZnO}$ thin films; effects of high energy electron irradiation and annealing // Nucl. Instr. and Meth. in Phys. Res. Sec. B, 266(9), p. 2021-2026 (2008). 
11. A. Ievtushenko, V. Karpyna, G. Lashkarev et al., Multilayered $\mathrm{ZnO}$ films of improved quality deposited by magnetron sputtering // Acta Physica Polonica, 114(5), p. 1131-1137 (2008).

12. A.I. Ievtushenko, G.V. Lashkarev, V.I. Lazorenko, V.A. Karpyna, M.G. Dusheyko, V.M. Tkach, L.A. Kosyachenko, V.M. Sklyarchuk et al., Effect of nitrogen doping on photoresponsivity of $\mathrm{ZnO}$ films // physica status solidi (a), 207(7), p. 1746-1750 (2010).

13. J.H. Jou, M.Y. Han, D.J. Cheng, Substrate dependent internal stress in sputtered zinc oxide thin films // J. Appl. Phys. 71, p. 4333-4336 (1992).

14. R. Cebulla, R. Wendt, K. Ellmer, Al-doped zinc oxide films deposited by simultaneous rf and dc excitation of a magnetron plasma: Relationships between plasma parameters and structural and electrical film properties // J. Appl. Phys. 83(2), p. 1087 (1998).

15. J. Hinze, K. Elmer, In situ measurement of mechanical stress in polycrystalline zinc-oxide thin films prepared by magnetron sputtering // J. Appl. Phys. 88(5), p. 2443-2450 (2000).

16. I. Shtepliuk, G. Lashkarev, V. Khomyak et al., Features of the influence of the deposition power and $\mathrm{Ar} / \mathrm{O}_{2}$ gas ratio on the microstructure and optical properties of the $\mathrm{Zn}_{0.9} \mathrm{Cd}_{0.1} \mathrm{O}$ films // Thin Solid Films, 520(14), p. $4772-4777$ (2012).

17. C.R. Aita, R.J. Lad, T.C. Tisone, Effect of $\mathrm{rf}$ power on reactively sputtered zinc oxide // J. Appl. Phys. 51(12), p. 6405-6410 (1980).
18. B. Szyszka, Transparent and conductive aluminum doped zinc oxide prepared by mid-frequency reactive magnetron sputtering // Thin Solid Films, 351, p. 164-169 (1999).

19. S. Jäger, B. Szyszka, J. Szczyrbowski, G. Bräuer, Comparison of transparent conductive oxide thin films prepared by a.c. and d.c. reactive magnetron sputtering // Surf. Coatings Technol. 98(1-3), p. 1304-1314 (1998).

20. N. Gopalakrishnan, B.C. Shin, H.S. Lim, G.Y. Kim, Y.S. Yu, Comparison of $\mathrm{ZnO}: \mathrm{GaN}$ films on $\mathrm{Si}(111)$ and $\mathrm{Si}(100)$ substrates by pulsed laser deposition // Phys. B: Condens. Matter, 376-377, p. 756-759 (2006).

21. W. Water, S.-Y. Chu, Physical and structural properties of $\mathrm{ZnO}$ sputtered films // Mater. Lett. 55, p. 67-72 (2002).

22. J.A. Thornton, D.W. Hoffman, Stress-related effects in thin films // Thin Solid Films, 171, p. 531 (1989).

23. B.D. Cullity, Elements of X-Ray Diffraction. Addison-Wesley, 1956, p. 431-453.

24. T. Minami, Present status of transparent conducting oxide thin-film development for Indium-Tin-Oxide (ITO) substitutes // Thin Solid Films, 516(17), p. 5822-5828 (2008).

25. T. Minami, H. Nanto, S. Takata, Highly conductive and transparent zinc oxide films prepared by $\mathrm{rf}$ magnetron sputtering under an applied external magnetic field // Appl. Phys. Lett. 41(10), p. 958960 (1982). 